\title{
AN ALGEBRAIC CHARACTERIZATION OF COMPLETE INNER PRODUCT SPACES
}

\author{
VASILE I. ISTRATESCU \\ Universitat Konstz \\ Fakultat fur Mathematik \\ Posfach 5560 \\ D-7750 KONSTANZ 1 \\ (Received October 1, 1983)
}

ABSTRACT. We present a characterization of completc initer product spaces using an involution on the set of all bounded linear operators on a Banach space. As a metric conditions we impose a "multiplicative" property of the norm for hermitain operators. In the second part we present a simpler proof (we believe) of the Kakutani and Mackney theorem on the characterizations of complete inner product spaces. Our proof was suggested by an ingenious proof of a similar result obtained by $N$. Prijatelj.

KEY WORDS AND PHRASES. COMriete inner product spaces, an algebraic characterization, hermitian projection, Kakutani-Mackey's iheirem.

1980 AMI SUBJIC'CI CLASSIFICATION CODE. $46 E 20$.

0 . INTRODUCTION. Let $X$ be a complex Banach space and $L(X)$ be the set of all bounded linear operators on $X$. There are conditions about $L(X)$ (or parts of $L(X)$ ) which force $X$ to be an inner product space,i.e. these conditions imply the existence of an inner product $<,>$ on $X$ such that for all $x$ in $x$,

$$
\|x\|^{2}=<x, x>
$$

where $\|$,$\| denotes the norm of the Banach space x$.

One of these sets of conditions was formulated by S.Kakutani and G.W.Mackey $(1944,1946)$ and reads as follows:suppose that on $L(X)$ we have a mapping " * whose values are in $L(X)$ such that the following relations hold:

1. $(\mathrm{T}+\mathrm{S}) *=\mathrm{T}^{*}+\mathrm{S}^{*}$,

2. $\left(\mathrm{T}^{*}\right) *=\mathrm{T}$,

3. $(\mathrm{TS})^{*}=\mathrm{S}^{*} \mathrm{~T}^{*}$,

4. $\mathrm{T}^{*} \mathrm{~T}=\mathrm{O}$ then $\mathrm{T}=\mathrm{O}$.

N.Prijatelj (1964) has considered the following set of conditions: on $L(X)$ there exists a map " * " whose values are in $L(X)$ and such that the following relations hold:

$$
\text { 1. }(T+S) *=T^{*}+S^{*},
$$


2. $\left(\mathrm{T}^{*}\right) *=\mathrm{T}$,

3. $(T S) *=S^{*} T *$,

4. $\|\mathrm{T} * \mathrm{~T}\|=\|\mathrm{T}\|^{2}=\|\mathrm{T} *\|^{2}$.

In what follows we present a new set of conditions on $L(X)$ which imply the existence of an inner product on $X,<$, > satisfying the relation

$$
\|x\|^{2}=\langle x, x\rangle
$$

and which simplifies to some extent the conditions imposed by N.Prijatelj.

Further we present a new proof of the Kakutani-Mackey theorem which we believe to be simpler than the original one.Our proof is inspired from the proof in the Prijatelj's paper.

1. AN ALGEbraic Characterization OF COMPLETE INNER PRODUCt SPACES.

Let $X$ be a complex Banach space and $L(X)$ be the set of all bounded linear operators on $X$.

Theorem 1.1.Suppose that on $L(X)$ there exists a mapping " *" with values in $L(X)$ such that the following conditions are satisfied:

1. $(T+S)^{*}=T^{*}+S^{*}$,

2. $\left(T^{*}\right) *=T$,

3. $(\mathrm{TS}) *=\mathrm{S} * \mathrm{~T} *$

4. if $P$ is a hermitian projection (i.e., $P^{2}=P=P *$ ) then

$$
\|\mathrm{P}\|^{2}=\left\|\mathrm{P}^{2}\right\| \text {. }
$$

Then on $X$ there exists an inner product space $<,>$ with the property that

$$
\|x\|^{2}=<x, x>\text {. }
$$

Proof.Let us consider $a, b$ be two arbitrary linearly independent elements in $x$ which generate a (closed) subspace of $X$, which we denote by $x_{2}$.

We consider an element $f$ in $X$ which has the followina pronerties:

$$
\begin{aligned}
& \text { 1. } f(a)=\|a\| \\
& \text { 2. }\|f\|=1 .
\end{aligned}
$$

Using $f$ we define the following operator $p$, by the formula:

$$
\mathrm{P}_{1} \mathrm{x}=\mathrm{f}(\mathrm{x}) /\|\mathrm{a}\| \cdot \mathrm{a} \text {. }
$$

It is not difficult to see that this operator is a hermitian projection.

Now since

$$
b=P_{1} b+\left(I-P_{1}\right) b
$$


we get that $\left(I-P_{1}\right) b \neq 0$ (since $a, b$ are supposed linearly independent). We choose $g$ in with the properties:

$$
\begin{aligned}
& \text { 1. } g(b)=\|b\|, \\
& \text { 2. }\|g\|=1 .
\end{aligned}
$$

We consider the following operator on $x$ :

$$
P_{2} x=g(x) /\left\|\left(I-P_{1}\right) b\right\| \cdot\left(I-P_{1}\right) b \text {. }
$$

This is a hermitain projection and we have the relations:

$$
\mathrm{p}_{1} \mathrm{P}_{2}=\mathrm{P}_{2} \mathrm{P}_{1}=\mathrm{O}
$$

which implies that the operator $\mathrm{P}$ defined by the formula

$$
\mathrm{P}=\mathrm{P}_{1}+\mathrm{P}_{2}
$$

is a hermitian projection on $X$. From our condition we get that the norm of $\mathrm{P}$ is 1. Then the Kakutani-Bohnenblust theorem implies that on the space $X$ there exists an inner product satisfying the theorem.

Remark 1.2 Our conditions in the theorem 1.1 are much like to those of Kakutani-Mackey and Prijatelj; the conditions 1-3 are identical while the condition 4 requires in fact that the Prijatelj's condition must be satisfied for hermitian operators which are projections.

2. A PROOF OF THE KAKUTANI-MACKEY'S THEOREM.

In what follows we present a proof of the Kakutani-Mackey's theorem using some ideas from the proof of N.Prijatelj's eleqant paper. In order to make the Note selfcontained we give here more details.

Let us consider an element $a \neq 0$ of $x$ and $f$ in $x *$ with the following properties :

$$
\begin{aligned}
& \text { 1. }\|f\|=1, \\
& \text { 2. } f(a)=\|a\| .
\end{aligned}
$$

We consider on $L(X)$ the following functional $g$ defined by the formula:

$$
g(T)=f(T a)
$$

and it is clear that this is a positive functional (i.e. $g(I)=1=\|$ l $\|$ ). Using this functional we define the following bilinear form on $L(X)$ (we remark that the positivity of $q$ implies that $g(T)=\left(q\left(T^{*}\right) *\right)$,

$$
\langle\mathrm{T}, \mathrm{S}\rangle=\mathrm{g}\left(\mathrm{S}^{*} \mathrm{~T}\right)
$$

which has all the properties of an inner product excent the fact that $\langle\mathrm{T}, \mathrm{T}\rangle=0$ does not necessarily implies that $\mathrm{T}=0$. Suppose now that the algebra has the following additional property: for any $u, v$ in , $\|\mathrm{u}\|=\|\mathrm{v}\|$ there exists a unitary operator $\mathrm{U}$ (i.e. $\mathrm{U}^{*} \mathrm{U}=\mathrm{UU}^{*}=\mathrm{I}$ ) such that $U u=v$. 
We consider now an operator defined on $L(X)$ with values in $X$ which is defined as follows:

$$
\mathrm{W}(\mathrm{T})=\mathrm{Ta} \text {. }
$$

Then it is clear that $\|W\|=\|a\|$ and if $N(W)$ is the null space of $W$ then $N(W)$ is a left ideal in $L(X)$. Another left ideal in $L(X)$ is defined as follows:

$$
\{R, R \in L(X),\langle R, R>=O\}=N \text {. }
$$

Then clear we have the inclusion

$$
\mathrm{N}(\mathrm{w}) \subset \mathrm{N}
$$

and we show that we have in fact an equality. Now since we suppose that $L(X)$ has the additional property with respect to unitary operators, we consider an arbitrary element $x$ of $x, x \neq 0$. Then we find an unitary operator $U$ with the property that

$$
\mathrm{x} /\|\mathrm{x}\|=\mathrm{Ua} \text {. }
$$

Since $\|x\| U$ is in $L(x)$ we get that

$$
\mathrm{W}(\|\mathrm{x}\| \mathrm{U})=\mathrm{x} \text {. }
$$

Now in order to prove the above equality we must prove the inclusion in the converse sense and for this it suffices to prove that

$$
\mathrm{C}_{\mathrm{N}(\mathrm{W})} \subset \mathrm{C}_{\mathrm{N}} \text {. }
$$

Suppose that $\mathrm{T}$ is in $\mathrm{C}_{\mathrm{N}(\mathrm{W})}$ and then

$$
\mathrm{W}(\mathrm{T})=\mathrm{Ta}=\mathbf{x} \neq 0 \text {. }
$$

We find a unitary operator $U$ in $L(X)$ with the property that

$$
\mathrm{x}=\|\mathrm{x}\| \mathrm{Ua}
$$

or

$$
\mathrm{Ta}=\|\mathrm{x}\| \mathrm{Ua}
$$

which gives that

$$
(T-\|x\| U) \quad a=0 .
$$

This implies that

$$
(\mathrm{T}-\|\mathrm{x}\| \mathrm{U}) \varepsilon \mathrm{N}(\mathrm{W}) \subset \mathrm{N} \text {. }
$$

Now if $T, S$ are in $L(X)$ and $T-S$ is in $N$ then

$$
g(T * T)=g(S * S) .
$$

Further this means that

$\left|g\left(T^{*} T\right)-g(S * S)\right|=|g(T *(T-S))+g((T-S) * S)| \leqq|g(T *(S-S))|+|g((T-S) * S)|$ and applying the Cauchy-Buniakowsky's inequality (for positive functionals) we get that

$$
\begin{aligned}
& g\left(T^{*}(T-S)\right)=0 \\
& g\left((T-S)^{*} S\right)=0 .
\end{aligned}
$$

Since $(T-\|x\| U)$ is in $N$ we have that 


$$
g\left(T^{*} T\right)=g(\|x\| U *\|x\| U)=\|x\|^{2} g\left(U^{*} U\right)=g(I)\|x\|^{2} .
$$

Thus if $x \neq 0, g\left(T^{*} T\right) \neq 0$ and thus we have that $T$ is in $C_{N}$ which implies the inclusion

$$
\mathrm{N} \subset \mathrm{N}(\mathrm{W})
$$

and thus the equality follows.

We form now the quotient space $L(X) / N(W)=L(X) / N$ and we remark that this is isomorphic to $X$, for each $x$ in $X$ the corresponding element in $L(X) / N$ is the equivalence class determined by the condition $W(T)=x$.

Using this isomorphism we can define an inner product on $X$ as follows: if $x, y$ are arbitrary elements in and $[x],[y]$ denotes the corresponding elements in $L(X) / N$ then we set

$$
\langle\mathrm{x}, \mathrm{y}\rangle=\mathrm{g}(\mathrm{u} \quad \mathrm{v})
$$

with $u$ arbitrary in [ $\mathrm{x}$ ] and $\mathrm{v}$ arbitrary in [y] .

From the Cauchy-Buniakowsky's inequality (for positive functionals) we see immediately that $<>$ is well defined in the sense that it does not depend on $u, v$ in the equivalence classes.

of fundamental importance of the above inner product is the property

$$
\langle x, x\rangle=\|x\|^{2} \text {. }
$$

Indeed, suppose that $W(T)=\|x\|$ and thus

$$
\langle\mathrm{x}, \mathrm{x}\rangle=\mathrm{g}\left(\mathrm{T}^{*} \mathrm{~T}\right)=\|\mathrm{x}\|^{2}
$$

and the assertion follows.

In order to complete the proof of the Kakutani-Mackey's theorem we must show that $L(X)$ satisfies the additional oroperty formulated above.

Let us consider a two-dimensional subspace of $x$ generated by the linearly independent elements $e_{1}$ and $e_{2}$. We may suppose without loss of generality that $\left(e_{1}, e_{2}\right)$ satisfies the following properties:

$$
\begin{aligned}
& \text { 1. }\left\|e_{i}\right\|=1, i=1,2 \text {, } \\
& \text { 2. for some fixed } f \text { in } x^{*}, f\left(e_{1}\right)=1, f\left(e_{2}\right)=0 \text {. }
\end{aligned}
$$

Suppose now that $x$ is an arbitrary element of $x_{2}$, the subspace generated by $e_{1}$ and $e_{2}$. We may suppose also that the norm of $x$ is 1 .

Then

$$
\mathrm{x}=\mathrm{Ae}{ }_{1}+\mathrm{Be}_{2}
$$

where A, B are scalars. 
We must prove that there exists a unitary operator $U$ with the property that

$$
\mathrm{Ue}_{1}=\mathrm{x} \text {. }
$$

Now, every operator on $x_{2}$ renresented by a $2 \times 2$ matrix

$$
\left(\begin{array}{ll}
a_{11} & a_{12} \\
a_{21} & a_{22}
\end{array}\right)
$$

and the adjoint by the matrix

$$
\left(\begin{array}{ll}
\tilde{a}_{11} & \tilde{a}_{12} \\
\tilde{a}_{21} & \tilde{a}_{22}
\end{array}\right)
$$

Since the property 1 holds the elements $a_{i j}$ are linear combinations of the conjugate, i.e.,

$$
a_{i j}=\sum_{k} b_{i j}^{i k} \bar{a}_{k j} \text {. }
$$

Further, since the properties 2 and 3 holds, taking into account that the identity operator is hermitian and that the operator

$$
P_{1} x=f(x) e_{1}
$$

is hermitian and a projection as well as the fact that for any operator $\mathrm{T}, \mathrm{T}^{*} \mathrm{~T}$ is hermitian, we get that the coefficients satisfy the following relations:

$$
\begin{aligned}
& 1=b_{11}^{12}+b_{11}^{22}, \circ=b_{12}^{11}+b_{21}^{22} \\
& \mathrm{o}=\mathrm{b}_{21}^{11}+\mathrm{b}_{21}^{22} \quad, \quad 1=\mathrm{b}_{22}^{11}+\mathrm{b}_{22}^{22} \\
& 1=b_{11}^{11}, \quad, o=b_{12}^{11}, \quad, \quad=b_{21}^{11}, o=b_{22}^{11} \text {, } \\
& b_{11}^{21}=b_{12}^{12}=b_{11}^{12}=b_{11}^{21}=b_{22}^{12}=b_{22}^{21}=0 \text {, } \\
& \mathrm{b}_{12}^{21}=\mathrm{b}_{12}^{21}=r, \mathrm{r} \text { a real number, } \\
& \mathrm{b}_{21}^{12}=\mathrm{r}^{-1} \text {. }
\end{aligned}
$$

Thus we have

$$
\mathrm{a}_{11}=\overline{\mathrm{a}}_{11}, \mathrm{a}_{12}=\overline{\mathrm{a}}_{21}, \mathrm{a}_{21}=\overline{\mathrm{a}}_{12}, \mathrm{a}_{22}=\overline{\mathrm{a}}_{22}
$$

(where the bar denotes the conjugate).

Now we use the fact that for unitary operators $U$ the relations must agrre again and thus the following relations must be hlod:

$$
\begin{aligned}
& a_{11} \bar{a}_{11}+r a_{21} \bar{a}_{21}=1=a_{11} \bar{a}_{11}+r^{-1} a_{12} \bar{a}_{12}, \\
& a_{12} \bar{a}_{11}+r a_{22} \bar{a}_{21}=0=r a_{11} \bar{a}_{21}+a_{12} \bar{a}_{22}, \\
& r^{-1} a_{11} \bar{a}_{12}+a_{21} \bar{a}_{22}=0=a_{21} \bar{a}_{11}+r^{-1} a_{22} \bar{a}_{12} \\
& r^{-1} a_{12} \bar{a}_{12}+a_{22} \bar{a}_{22}=1=r a_{21} \bar{a}_{21}+a_{22} \bar{a}_{22} .
\end{aligned}
$$


In this case we must have

$$
a_{22} \bar{a}_{22}=a_{11} \bar{a}_{11}, a_{12} \bar{a}_{12}=r\left(1-a_{11} \bar{a}_{11}\right)=r^{-1}\left(1-a_{11} \bar{a}_{11}\right) \text {. }
$$

Thus if we set

we have that

$$
a_{11}=r e^{i \theta}
$$

$$
\begin{aligned}
& a_{22}=-a e^{i(\theta-s+t)}, a_{12}=\left(\left(1-a^{2}\right) r\right)^{1 / 2} e^{i(\theta+t)} \\
& a_{21}=\left(\left(1-a^{2}\right) r^{-1}\right)^{1 / 2} e^{i(\theta-s)}
\end{aligned}
$$

where, $s, t$ are neal numbers (parameters).

Thus the matrix representing a unitary operator has the form:

$$
\left(\begin{array}{cc}
a e^{i \theta} & \left(\left(1-a^{2}\right) r\right)^{1 / 2} e^{i(\theta+s)} \\
\left(\left(1-a^{2}\right) r^{-1}\right) 1 / 2 e^{i(\theta-t)} & -a e^{i(\theta-s-t)}
\end{array}\right)
$$

Now if $x$ is an arbitrary element of $x_{2}$ (of norm 1) we must show that for some unitary operator $U, U e_{1}=x$. This clear is equivalent with the relation

Now if

$$
a e^{i \theta} e_{1}+\left(1-a^{2}\right)^{1 / 2} e^{i(\theta-t)}=A e_{1}+B e_{2}=x
$$

from

$$
A=a_{1} e^{i \theta} 1, B=a_{2} e^{i \theta} 2
$$

$$
A / B=\left(a_{1} / a_{2}\right) \cdot e^{i}\left(\theta_{1}-\theta_{2}\right)=\left(1-a^{2}\right)^{1 / 2} a^{-1} e^{-i t}
$$

we conclude that

and thus

$$
\theta_{1}-\theta_{2}=t, a=\left(a_{1}^{2} /\left(a_{1}^{2}+a_{2}^{2}\right)\right.
$$

$$
x=C\left(a e^{i \theta_{1}} \cdot e_{1}+\left(1-a^{2}\right)^{1 / 2} e^{i\left(\theta_{1}-\theta_{2}\right)} \cdot e_{2} .\right.
$$

From the fact that the norm of $x$ is 1 we conclude that $a=a_{1}$, $a_{2}=\left(1-a^{2}\right)^{1 / 2}$. Thus the matrix of the unitary operator is

$$
\left(\begin{array}{cc}
A e^{i \theta} 1 & \left(1-A^{2}\right)^{1 / 2} e^{i(\theta+s)} \\
\left(1-A^{2}\right)^{1 / 2} e^{i(\theta-t)} & -A e^{i(\theta+t-s)}
\end{array}\right) .
$$

Thus the additional property holds for our algebra and this completes the proof of the theorem. REFERENCES

1. KAKUTANI S. and MACKEY, G.W., Two characterization of real Hilbert spaces. Ann. Math. 45 (1944) 50-58.

2. KAKUTANI S., Ring and lattice characterizations of complex Hilbert spaces. Bu11. Amer. Math. Soc. 52 (1946) 727-733.

3. PRIJATELJ, NIKO, Algebra characterizations of Hilbert spaces. Publications of the Department of Mathematics, I. Ljubljana, 1964 pp43-57.

4. ISTRATESCU, V.I., Inner product structures. Theory and applications. D. Reidel Pub1. Co. Dordrecht-Boston. (To appear). 


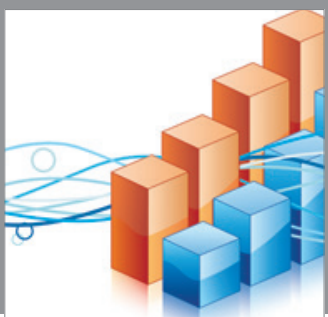

Advances in

Operations Research

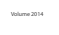

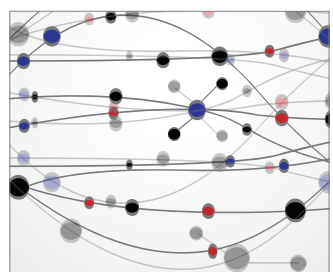

\section{The Scientific} World Journal
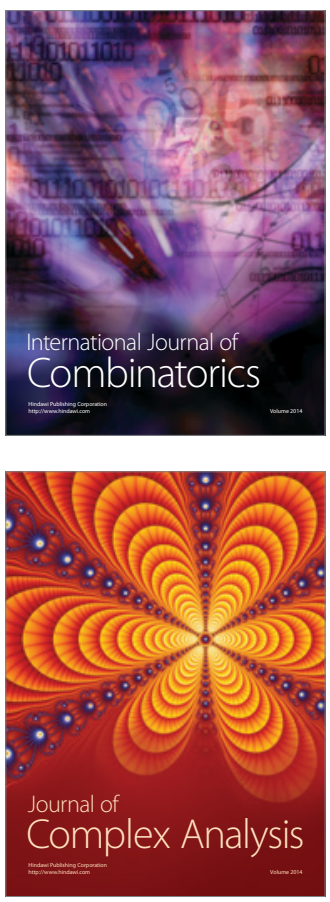

International Journal of

Mathematics and

Mathematical

Sciences
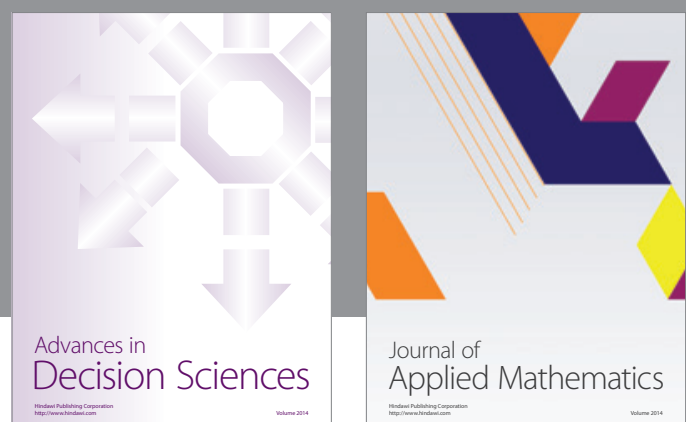

Journal of

Applied Mathematics
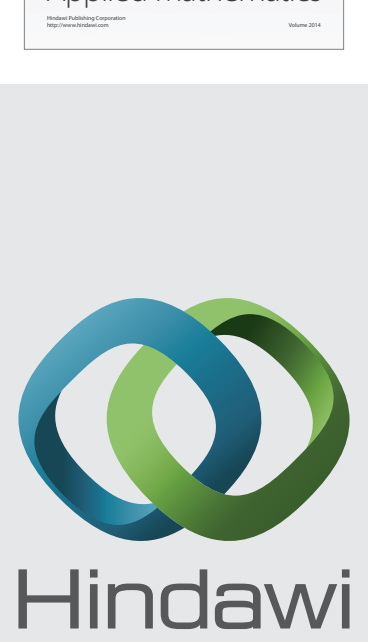

Submit your manuscripts at http://www.hindawi.com
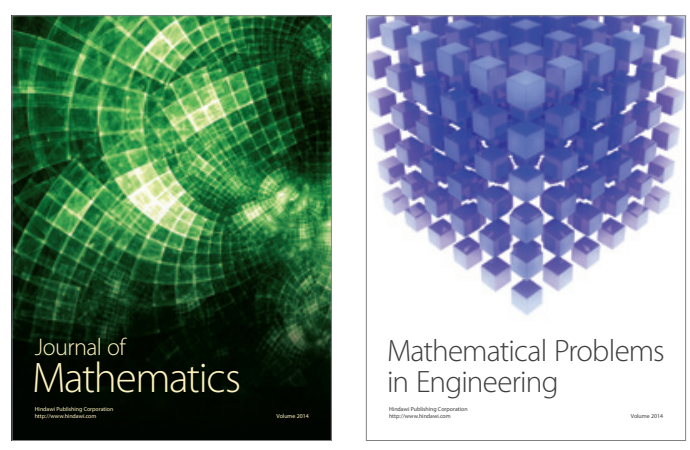

Mathematical Problems in Engineering
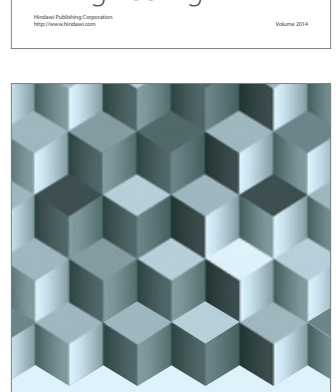

Journal of

Function Spaces
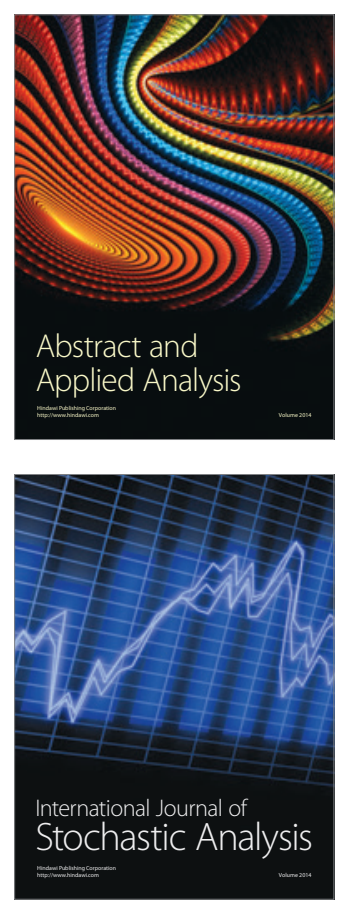

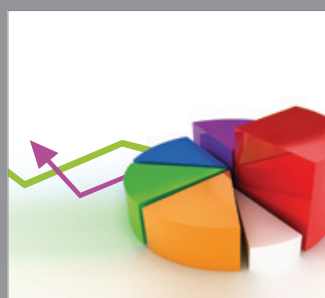

ournal of

Probability and Statistics

Promensencen
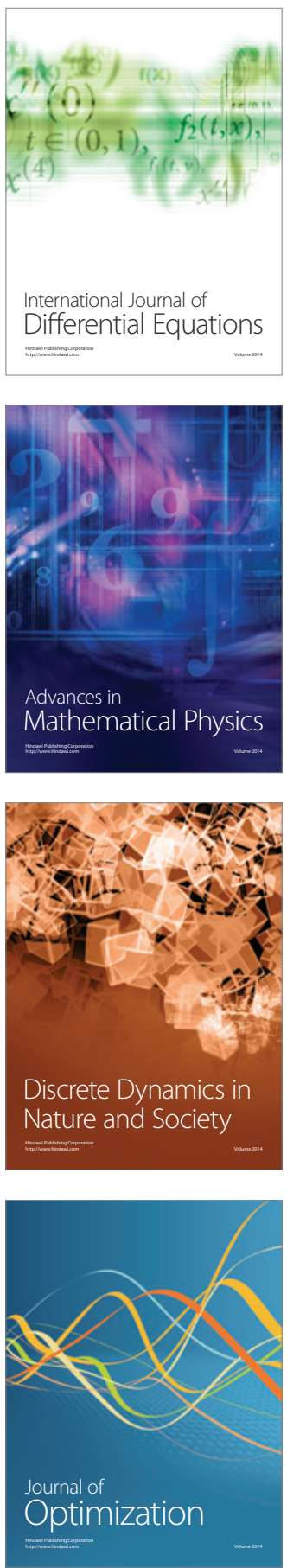